\title{
Linden, wichtige Nutz- und Ziergehölze mit langer kulturhistorischer Tradition
}

\author{
Veit M. Dörken, Hilke Steinecke \& Ilse Zündorf
}

\begin{abstract}
Small-leaved lime (Tilia cordata) and large-leaved lime (Tilia platyphyllos) are native trees in central Europe. They are used as forest trees and also as ornamental trees. Planting lime trees in village centers, on farms and manors or next to chapels has a long tradition. Lime-trees are popular for the sweet fragrance of the flowers and the honey. The paper introduces the biology and the cultural history of lime trees. Due to its cultural importance in central Europe the small-leaved lime was elected as tree of the year 2016. The large-leaved lime has already been tree of the year 1991.
\end{abstract}

\section{Zusammenfassung}

Winter- und Sommer- Linden sind bei uns heimische Bäume. Diese sowie andere Linden sind wichtige Nutz- und Ziergehölze. Das Pflanzen von Linden im Zentrum von Dörfern, an Höfen oder an Kapellen hat bei uns eine lange Tradition. Beliebt sind Linden nicht nur wegen ihres süßen Blütenduftes, dem von ihnen stammenden milden Lindenhonig oder der Verwendung als Heilpflanze. Die Biologie verschiedener Linden-Arten, ihre Nutzung, Kulturgeschichte sowie das Hummelsterben unter Linden werden angesprochen. Wegen ihrer großen kulturellen Bedeutung in Mitteleuropa wurde die Winter-Linde (Tilia cordata) zum Baum des Jahres 2016 gekürt. Die Sommer-Linde (Tilia platyphyllos) war bereits 1991 Baum des Jahres.

\section{Linden an vielen Orten}

Verschiedene Linden-Arten begegnen uns häufig als Straßen- und Hofbäume, auf Friedhöfen oder an Kapellen und Kirchen sowie natürlich auch als heimische Gehölze in der Natur. Linden werden seit Jahrhunderten gepflanzt, was sicherlich auch daran liegt, dass sie nicht nur wichtige Nutzhölzer sind, sondern auch einen großen Symbolwert haben. In Deutschland werden neben den heimischen auch zahlreiche fremdländische Arten und deren Sorten sowie Hybriden gepflanzt (z.B. Jablonski 2011, Jablonski \& Plietzsch 2013, 2014). Linden können mehrere Hundert, mitunter über 1000 Jahre alt werden. Derartige knorrige, meist hohle Baumveteranen ziehen schon lange die Aufmerksamkeit auf sich und sind meist mit bestimmten Geschichten verbunden. Linden werden nicht selten auch in Musik, Gedichten und Märchen thematisiert, so beispielsweise auch in dem bekannten Kindermärchen „Klingt meine Linde“ von Astrid Lindgren.

Jahrhundertealte Linden findet man über ganz Deutschland verteilt. Einige der besonders alten und imposanten Winter-Linden (Tilia cordata) sind z. B. die Riesenlinde zu Heede, (Kreis Emsland), die Dorflinde in Speck (Kreis Mür-

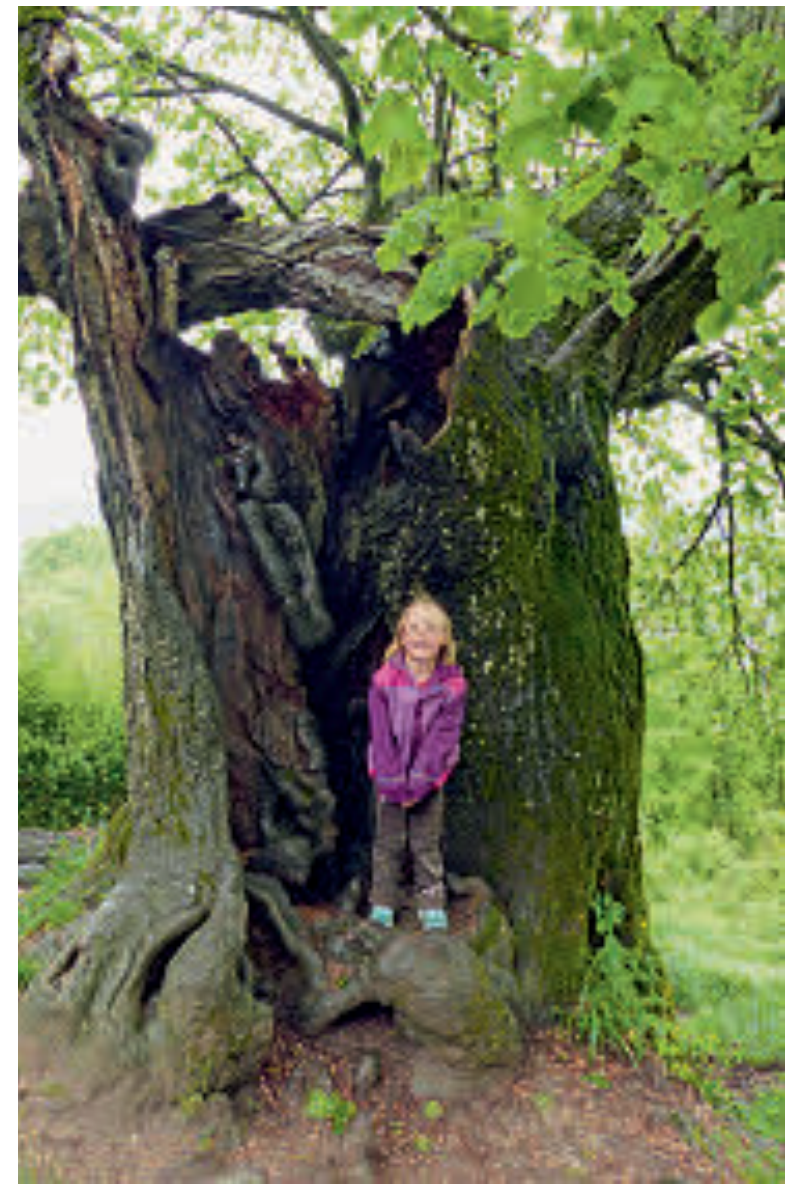

Abb. 1: Alte Linden sind oft hohl, dass man sich in ihnen verkriechen kann. Hier eine stattliche Linde am Haldenhof am Bodensee. 

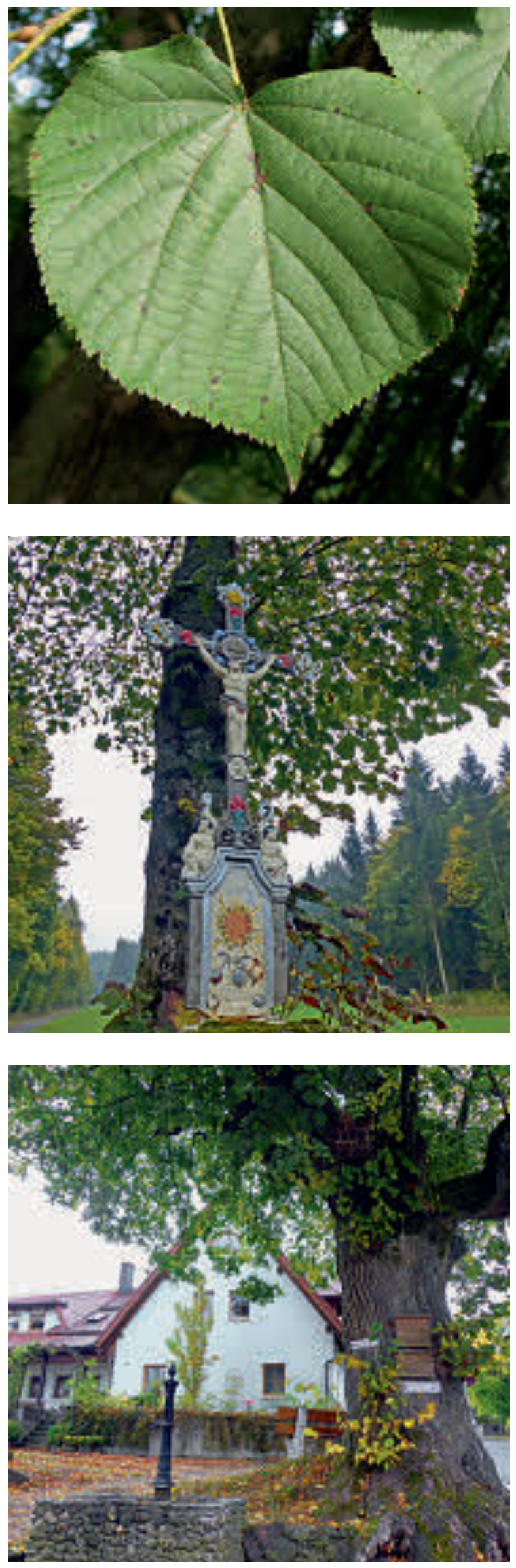

litz), die Linde zu Bermoll (Lahn-Dill-Kreis), die Zwölf-Apostel-Linde in Gehrden (Kreis Höxter) oder auch die Tassilolinde bei Wessobrunn (Kreis Weilheim-Schongau). Besonders alte Sommer-Linden (Tilia platyphyllos) sind z. B. die Linde in Asbeck (Kreis Borken), die Kaiser-Lotar-Linde in Königslutter (Kreis Helmstedt), die Linde in Steinbergkirche (Kreis Schleswig-Flensburg), die Schwedenlinde in Brielow (Kreis Potsdam-Mittelmark), die Friedhofslinde in Collm (Kreis TorgauOschatz) sowie die Tanzlinde in Himmelsberg (Kreis Marburg-Biedenkopf, FröHLICH 1989, KüHN et al. 2004).

Weitere der bemerkenswertesten, besonders großen und alten Linden in Deutschland sind in der Championtree-Liste der Deutschen Dendrologischen Gesellschaft e.V. aufgeführt. Über ganz Deutschland verteilt kann man alte, bemerkenswerte und Jahrhundertealte Linden finden (FröHLich 1989, KüHN et al. 2004).

\section{Linden, Bäume der Götter und des Gerichtes}

Schon in der Antike wurden Linden verehrt und mit den Göttern in Verbindung gebracht. Sie kommen in verschiedenen Mythen und Legenden vor. Das berühmte Paar Philemon und BAUcis wurde von den Göttern in eine Eiche und eine Linde verwandelt, um auch nach dem Tod noch eng umschlungen nebeneinander existieren zu können (Fischer-Rizzi 1996).

Auch den Kelten und Germanen war die Linde ein göttlicher Baum. Wegen ihrer herzförmigen Blätter und der bei vielen Exemplaren zudem herzförmigen Krone gilt die Linde als Baum der Liebe. In der Linde verehrten die Germanen deshalb die Erdmutter FreYA, auch Göttin des Glückes und der Liebe. Es gab alte

Abb. 2 (oben): Herzförmiges Blatt einer Winter-Linde (Tilia cordata).

Abb. 3 (Mitte): Bildstock an einer Linde in Oberfranken.

Abb. 4 (unten): Dorflinde mit Dorfbrunnen in Engelhardsberg, Oberfranken.

Abb. 5 (Seite 107): Dorfkirche in Thethi (Albanische Alpen) mit drei alten Linden. 


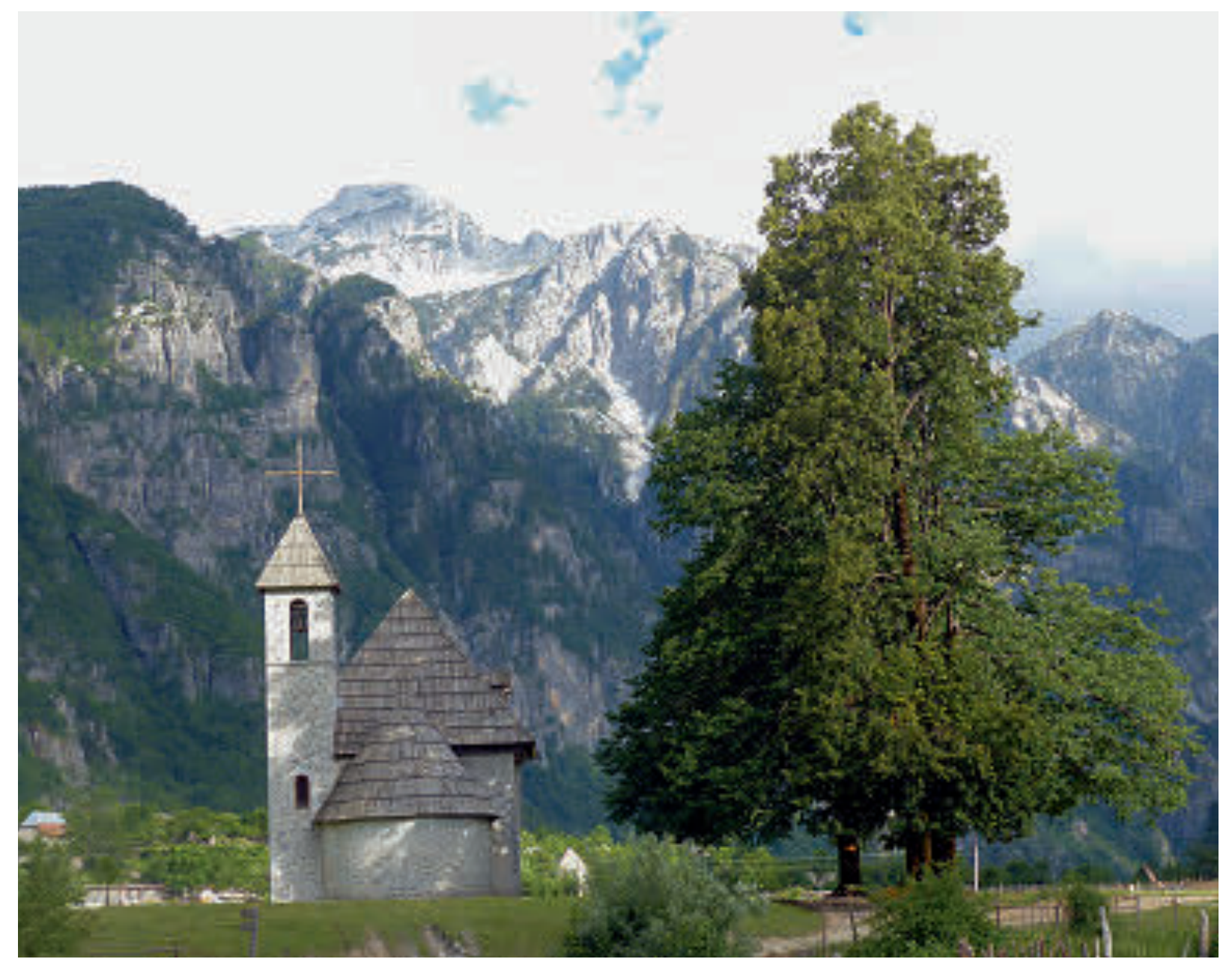

Linden, unter denen Thing-Versammlungen abgehalten wurden und Richter ihr Urteil sprachen. Die Linde konnte Urteile mitunter mild stimmen, denn an der Linde ist alles weich und mild, wie auch das Holz und der Honig (Schneebeli-Graf 2008). Der Name unseres Wochentages Dienstag ist auf den germanischen Gott Tyr (Beschützer des Things, latinisiert Mars thingsus) zurückzuführen. Am Gerichtstag, dem Thing-Tag (also dem Dienstag), wurde ein Verurteilter thingfest (dingfest) gemacht und bei einem sehr schlimmen Vergehen sogar an der Dorflinde aufgehängt.

In der Siegfried-Sage wurde ein Lindenblatt dem Helden zum Verhängnis. SiEgFried badete sich im Blute des von ihm getöteten Drachen und wurde dadurch unverwundbar. Unglücklicherweise fiel ihm dabei ein Lindenblatt zwischen die Schulterblätter, sodass er dort verwundbar blieb. Dies führte später zu seinem Tod.

\section{Vom heidnischen Götterbaum zum Marienbaum}

Viele Bäume, die von Kelten und Germanen früher als heilig verehrt wurden, haben bis heute auch im Christentum eine symbolische Bedeutung. Denn für die Kirchenvertreter war es mit der Einführung des christlichen Glaubens viel einfacher, die Bäume umzuwidmen und z. B. Gott oder Maria zuzuordnen, als die alte heidnische Verehrung dieser Bäume gänzlich $\mathrm{zu}$ verbieten und damit auszulöschen, indem markante alte Kult-Bäume (z. B. die Donar-Eichen) einfach gefällt wurden. So wurden aus den Freya-Linden vielerorts Marien-Linden. Sie wurden früher und werden auch noch heute häufig in der Nähe von Kirchen oder Kapellen gepflanzt. In Bayern gibt es kaum eine Kapelle, an der nicht eine Linde steht (LAUDERT 2009). Vielleicht aus Respekt vor den schönen Bäumen mit ihrem schweren süßen Blütenduft hat manch eine Linde sogar ihre Kirche überlebt, 


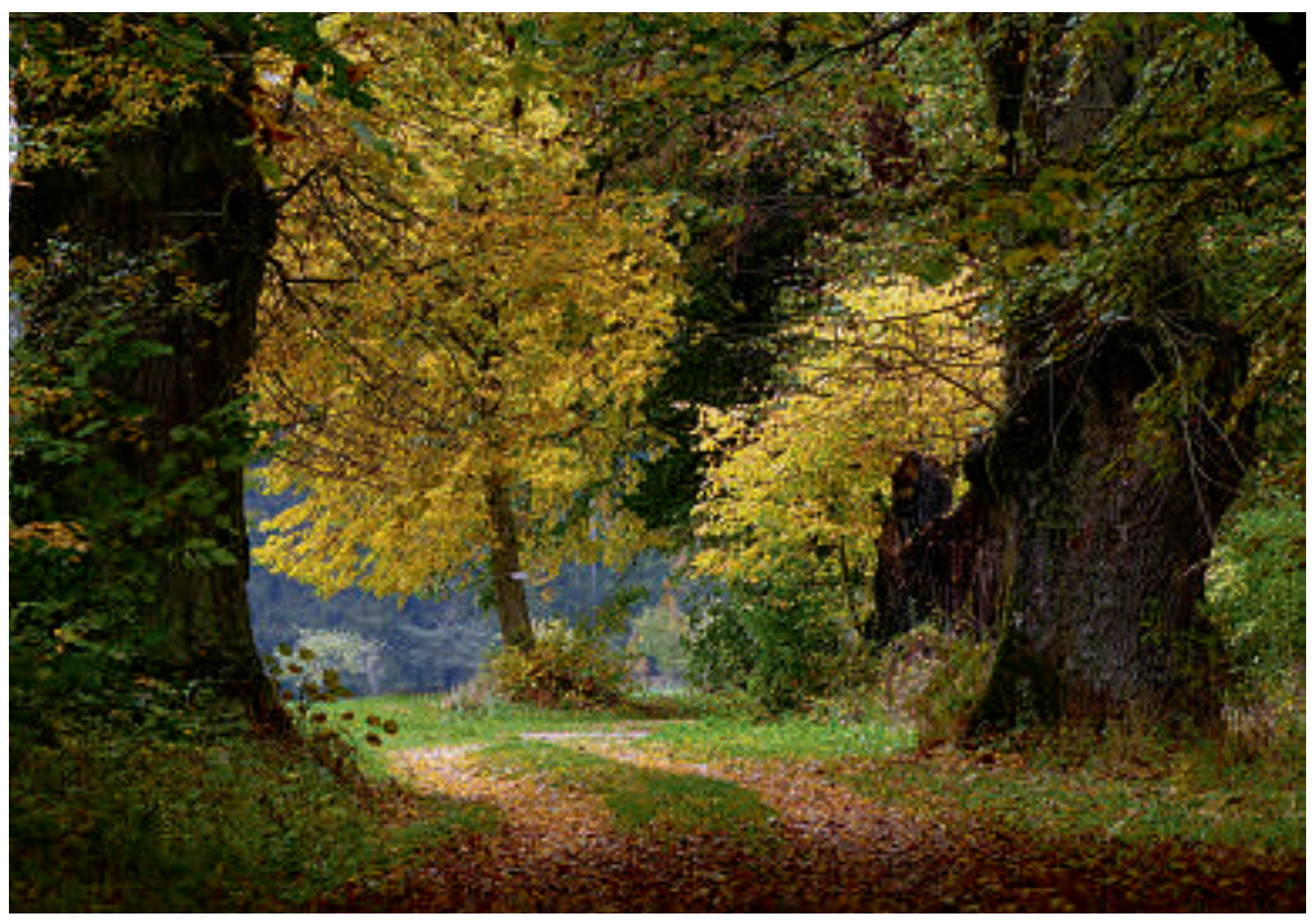

auch wenn diese aus verschiedensten Gründen zweckentfremdet oder zerstört wurde. So steht beispielsweise in Thethi in den Albanischen Alpen eine kleine hölzerne Dorfkirche, die von drei großen, alten Linden umgeben wird. Die Bäume sind alt; während der kommunistischen Ära in Albanien aber wurde die Kirche als Turnhalle benutzt und der Turm abgetragen. Heute ist die Kirche wieder erneuert und in den ursprünglichen Zustand versetzt. Mit ihren drei alten Linden wirkt sie romantisch schön gegen die schroffe Bergkulisse.

Linden galten früher als Symbol der (göttlichen) Unsterblichkeit und der unerschöpflichen Lebenskraft, was nicht zuletzt auf die scheinbare Unverwüstlichkeit der Bäume zurückzuführen ist, denn Linden überleben selbst schlimmste Kronenverletzungen, und Baumstubben treiben, wenn sie auf den Stock gesetzt wurden, später wieder willig aus (KüHN et al. 2004).

\section{Friedens-, Schutz- und Familienbäume}

Marien-Bäume galten natürlich auch als Schutzbäume für Haus und Hof, in deren Nähe sie früher häufig gepflanzt wurden. Nicht selten stehen noch heute alte Linden im Dorf neben dem Dorfbrunnen. „Am Brunnen vor dem Thore, da steht ein Lindenbaum: Ich träumt' in seinem Schatten so manchen süßen Traum“ ist die erste Strophe eines von Franz Schubert vertonten Gedichtes aus dem Zyklus „Winterreise“. Zum Gedenken an Pestopfer oder nach Friedensschlüssen wurden sogenannte Friedenslinden gesetzt. Die meisten der heute noch existierenden Friedenslinden gehen auf den Deutsch-Französischen Krieg (1870/71) zurück. Kaiser KarL DER Grosse hat in seiner Landgüterverordnung (capitulare de villis) angeordnet, dass vor jeder Hofstatt unbedingt ein Lindenbaum gepflanzt werden sollte als Schutzbaum vor Blitzschlag und bösen Geistern. Die Bäume sollten auch einzelnen Familienmitgliedern

Abb. 6 (links): Alte Lindenallee mit 400 Jahre alten Bäumen am Schloss Wiesentfels (Landkreis Bayreuth).

Abb. 7 (rechts): Alter Lindenhain im Schlosspark von Schloss Pretzfeld in der Fränkischen Schweiz. 


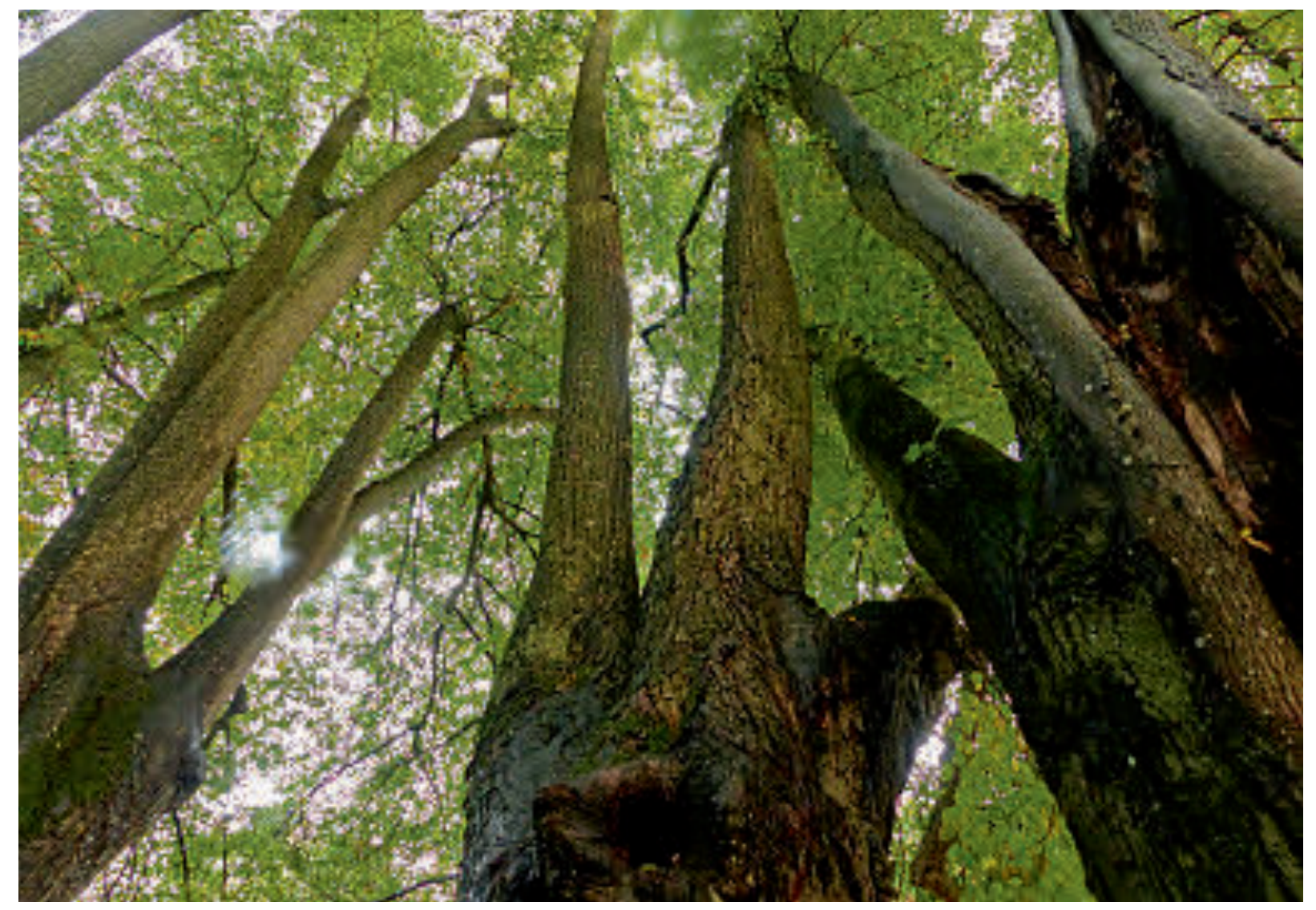

Glück bringen, vor allem wenn sie aus Anlass ihrer Geburt gepflanzt wurden. Auf der Insel Mainau im Bodensee beispielsweise beherrscht die imposante Victoria-Linde, eine SommerLinde, das Bild. Sie wurde vom Großherzog FrIEDRICH I. im Jahr 1862 anlässlich der Geburt seiner Tochter VICTORIA gepflanzt. Der Baum hat heute eine rund $20 \mathrm{~m}$ breite Krone, ist $25 \mathrm{~m}$ hoch bei einem Umfang von fast 4,9 Metern (FrÖHLICH 1989).

Besonders eindrucksvoll wirken Linden, wenn sie als Alleen gepflanzt sind. Alte, aber auch junge Lindenalleen findet man an Straßen, auf Friedhöfen sowie an alten Burg- und Schloss-Zufahrten. Der Rest einer Lindenallee mit bis zu 400 Jahre alten, knorrigen Bäumen kann am Schloss Wiesentfels im Landkreis Bayreuth bewundert werden. Einige der Bäume sind zwar in den letzten Jahren Stürmen zum Opfer gefallen, jedoch wurden die eindrucksvollen Stämme am Wegesrand liegen gelassen, sodass das Totholz nun neue Lebensräume für Pflanzen, Tiere und Pilze bildet. Aus Holzresten und Baumpilzen wurden trollähnliche
Figuren angefertigt, die der Wanderer hier unterwegs auf und neben den Bäumen entdecken kann. Viele Menschen der Umgebung fühlen sich mit der „einmal etwas anderen“ Lindenallee sehr verbunden. Die Pflege und Aufarbeitung dieser alten Lindenallee erhielt im Rahmen des Ortsverschönerungswettbewerbs 2011 einen Sonderpreis.

Linden haben zu jeder Jahreszeit ihren Reiz, ob es die zart belaubten Bäume im Frühling, die süß duftenden Blüten im Sommer, die leuchtend goldgelben Blätter im Herbst sind, oder ob es die gleichmäßige Wuchsform des kahlen Baumes ist. Unter Linden wurde geliebt und getanzt. Es gibt Tanzlinden, deren Äste durch Gestelle geleitet wurden und in denen sich dann eine Tanzplattform befand. Getanzt wurde aber auch auf dem Boden unter bestimmten Linden.

Viele Ortsnamen leiten sich von der Linde $a b$ (z. B. Linz, Lindau, Leipzig). Die Gemeinde Bodman-Ludwigshafen am Bodensee trägt in ihrem Stadtwappen drei Lindenblätter. Aber auch Lindau, Hohenlinden, Niederems, Selm, 

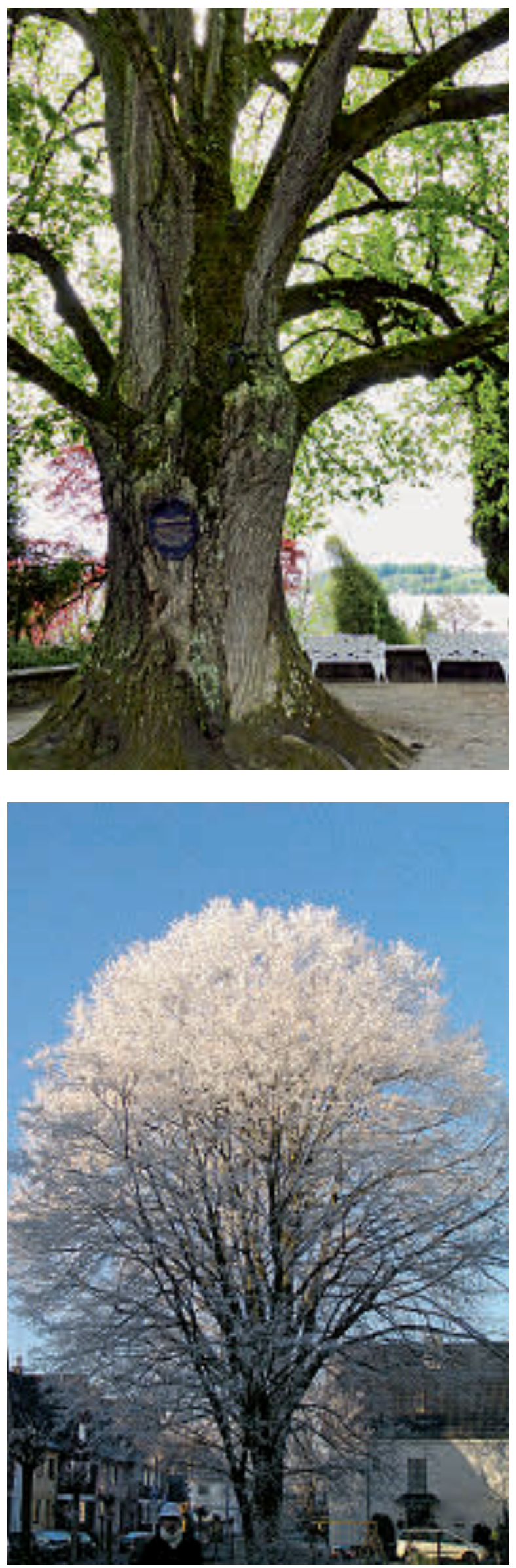

Großbettingen u.a. haben Lindenblätter oder Lindenbäume in ihrem Wappen verewigt. Geisenheim am Rhein in der Nähe von Rüdesheim und gleichzeitig das Tor zum Rheingau nennt sich explizit auch die Lindenstadt. Denn im Zentrum der kleinen Stadt steht am Marktplatz eine über 700-jährige Linde, die im Jahr 1568 erstmals erwähnt wird und im Mittelalter als Gerichtslinde diente. Wegen der Beliebtheit der Linden heißt auch die älteste Prachtstraße Berlins Unter den Linden. Die ersten Bäume ließ hier im Jahr 1647 der Kurfürst Friedrich Wilhelm pflanzen. Seit 1985 gehört die in Köln produzierte und in München spielende Serie „Lindenstraße“ zu den bekanntesten deutschen Fernsehserien.

Linde ist zudem ein weiblicher Vorname, der auch in Varianten wie z. B. Rosalinde, Lina oder Linda verwendet wird. Übersetzt bedeutet der Name die Freundliche oder die Schöne, Attribute, die auch dem Lindenbaum zugeordnet werden. In vielen Variationen findet sich die Linde in diversen Familiennamen wieder, nicht nur bei Udo Lindenberg und Astrid LindGREN. So geht auch der Familienname des berühmten Botanikers CARL v. Linné auf die Linde zurück. Den Namen hatte sich sein Vater, der zuvor wie in Schweden damals üblich keinen offiziellen Nachnamen hatte, selbst gegeben. Inspirieren lassen hatte er sich von einem dreistämmigen Lindenbaum in der Nähe seines Geburtshauses, dem Hof Jonsboda. So wie der Baum drei Hauptstämme hatte, nannten sich auch drei Familien nach diesem Baum, also außer Linné auch Lindelus und Tilian-

Abb. 8 (oben): Victoria-Linde auf der Insel Mainau im Bodensee.

Abb. 9 (unten): Rund 100 Jahre alte Silber-Linde in einer ebenso alten Dortmunder Vorortsiedlung. Wegen Leitungsarbeiten in der Straße wurden dieser schöne Baum sowie eine auf der gegenüberliegenden Straßenseite stehende, ebenfalls 100-jährige Winter-Linde 2014 gefällt. Bis zum Redaktionsschluss wurde an beiden Stellen nichts nachgepflanzt.

Abb. 10 (Seite 111): Ausschnitt aus dem Altar von Veit SToss in der Marienkirche in Krakau: Die Figuren des Altars, wie auch diese MARIA, bestehen aus Lindenholz. 
DER, beides Ableitungen von Bezeichnungen für die Linde. Nach der Linde benannt ist zudem das preußische Adelsgeschlecht vON DER Linden. Die noch heute existierende Familie hat Linien in Schlesien und Schweden.

\section{Nutzung und medizinische Bedeutung}

Linden sind vielseitig nutzbar, ob es nun Holz, Fasern, Blätter oder Blüten sind. Das Holz ist recht weich und eignet sich daher nicht als Bauoder Konstruktionsholz, jedoch hervorragend zum Schnitzen und Drechseln, aber auch als Blind-, Möbel- und Furnierholz. Ferner werden aus Lindenholz Holzschuhe und Musikinstrumente hergestellt. (Sснӥтt et al. 2002, LiebeRei \& Reisdorff 2007, Düll \& KutzelnigG 2011). Manch ein Altar, beispielsweise aus den Werkstätten von Tilmann Riemenschneider oder Veit Stoss, und viele Marienstatuen sind ebenfalls aus Lindenholz geschnitzt. Die Schwarze Madonna von Tschenstochau ist eine Mariendarstellung auf einer Lindenholztafel. Sie ist die heiligste und vermutlich auch bekannteste Mariendarstellung Polens. In früheren Zeiten wurde Lindenholz auch zur Herstellung von Holzkohle genutzt. Diese diente nicht nur zum Kochen und Heizen, sondern war zudem ein wichtiger Bestandteil von Schießpulver. Der Lindenbast wurde früher zur Herstellung von Flechtarbeiten wie z. B. Matten, Körben, Kleidungstücken und sogar Schuhen verwendet (Düll \& Kutzelnigg 2011). Beliebt ist heute der besonders milde Lindenblüten-Honig. Die Linde ist zudem eine traditionelle Heilpflanze. Ihre Blüten werden in der Volksmedizin wegen ihres Gehalts an Schleimstoffen, Flavonoiden, Gerbstoffen und ätherischem Öl als Tee bei Erkältungskrankheiten eingesetzt. Wissenschaftlich nachgewiesen sind diese Wirkungen allerdings nicht. Inwieweit die schweißtreibende Wirkung von Lindenblütentee tatsächlich auf die Inhaltsstoffe oder aber auf die mit dem Tee zugeführte heiße Flüssigkeit zurückzuführen ist, wird nach wie vor diskutiert (BLASCHEK 2007).

Wegen seiner vielseitigen Verwendung, kulturellen und ökologischen Bedeutung ist die Winter-Linde (T. cordata) zum Baum des Jahres

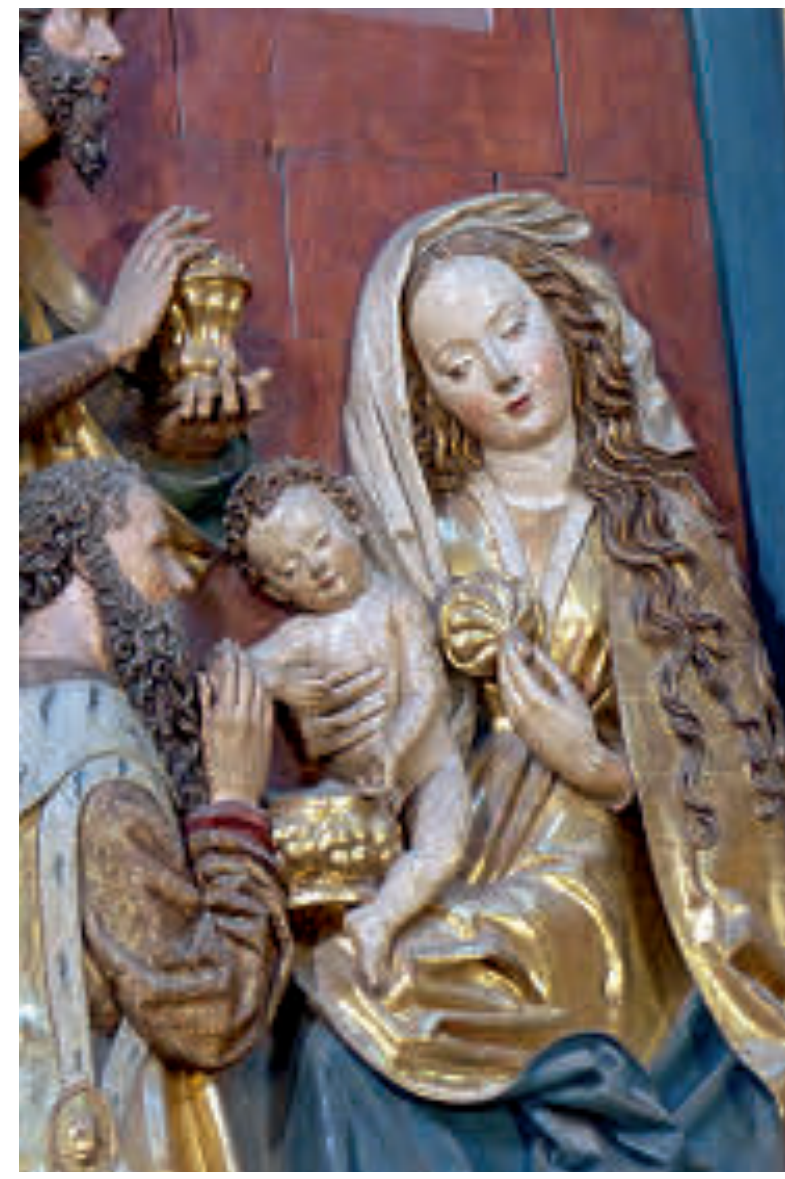

2016 auserwählt worden, die Sommer-Linde (T. platyphyllos) war Baum des Jahres 1991.

\section{Morphologie}

Linden sind sommergrün mit ausladenden Kronen und gehören zu den Reifholzbäumen, d.h. im Holz lässt sich der Kernbereich farblich nicht vom Splintbereich unterscheiden. Nicht selten sind die Stämme alter Bäumen hohl. Im Innenraum werden dann Sekundärwurzeln gebildet, die später nach Erreichen des Bodens zusätzlich der Stabilisierung, aber auch der Wasser- und Nährstoffaufnahme dienen (DüLl \& KutZeLNIGG 2011). Linden können sich somit quasi von innen heraus verjüngen und leben weiter, selbst wenn der Baum hohl ist und Teile der alten Krone bereits abgestorben sind. Ein altes Sprichwort besagt, dass Linden 300 Jahre kommen, 300 Jahre stehen und 300 Jahre vergehen.

Linden haben eine wechselständige und an den Seitenzweigen zweizeilig angeordnete Belaubung. Die Blätter sind meist deutlich herzförmig, bei einigen Arten mit einem asymmet- 

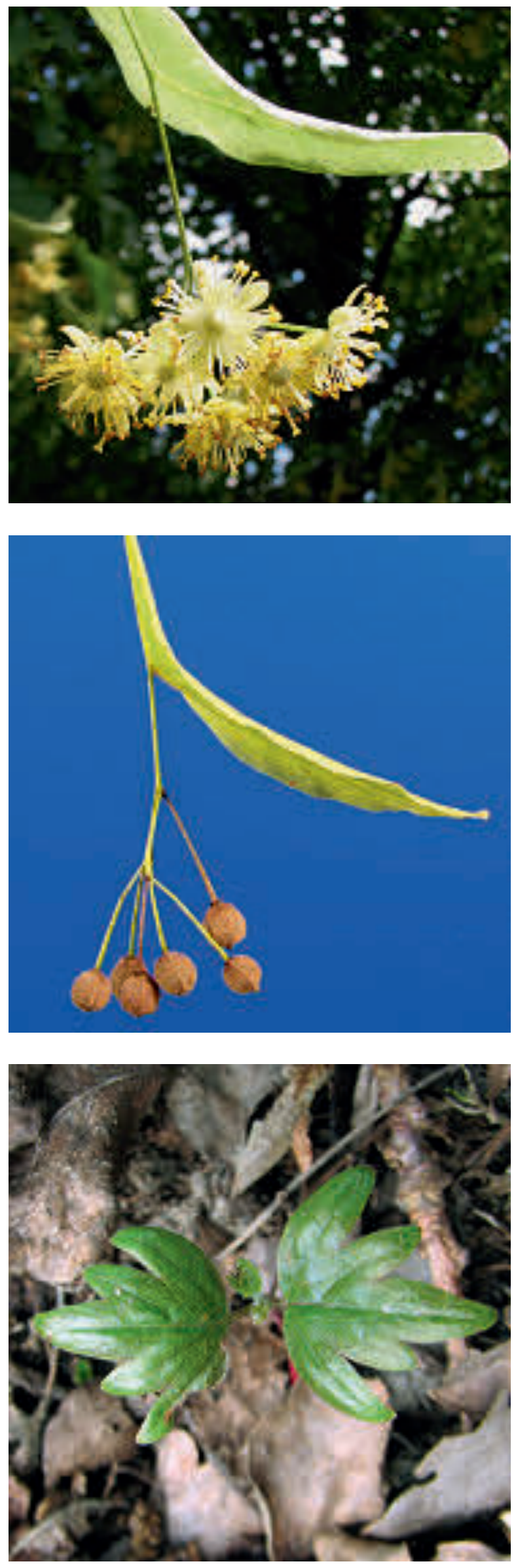

rischen Spreitengrund. Der Blattrand ist meist deutlich gesägt, bei manchen Linden wie z. B. $T$. henryana laufen die Blattnerven in kleine Grannenspitzen aus. Artspezifisch variiert die Behaarung der Blätter. Bei T. cordata werden z.B. in den Achseln der Hauptnerven auf der Blattunterseite braune Haarbüschel gebildet, bei T. platyphyllos sind sie weiß. Die Blattunterseite von T. tomentosa (Silber-Linde) ist vollständig weißfilzig behaart. Zwischen dem Laub kann man gelegentlich die parasitische Laubholz-Mistel (Viscum album) entdecken, die Linden wie auch weitere Laubgehölze wie z. B. Apfelbäume und Pappeln befällt.

Linden sind ausgesprochene Sommerblüher, wobei die Winter-Linde etwa zwei Wochen später als die Sommer-Linde blüht (Marzell 1979). Die aus China stammende T. henryana blüht erst im Juli oder August, wenn kaum noch ein anderer Baum blüht, was sie für die Verwendung als Ziergehölz sehr interessant macht. Die zahlreichen Einzelblüten (bei $T$. cordata 5-11, bei $T$. platyphyllos 2-5) sind in hängenden Blütenständen angeordnet. An ausgewachsenen Bäumen werden bis 60000 Einzelblüten angeordnet (Düll \& KutZelnigG 2011). Die Blüten- und später die Fruchtstände sind mittig auf ein lang ausgezogenes Vorblatt verlagert, das später als Flugorgan bei der Ausbreitung der Früchte durch den Wind dient. Die fünfzähligen, stark duftenden Blüten sind weiß bis cremeweiß gefärbt und produzieren Nektar, besonders in den Abend- und Nachtstunden (Düll \& KutZelnigg 2011). Es wird zudem reichlich Pollen ausgebildet, der ebenso eine wichtige Nahrungsquelle für Honigbienen darstellt (Düll \& KutZelnigg 2011). Die fünf Fruchtblätter bilden einen gemeinsamen Fruchtknoten, aus dem sich später eine etwa erbsengroße, schwach bis stark längsgerippte, kugelige Nussfrucht bildet. Die Fruchtschalen sind artspezifisch unterschiedlich dick. So kön-

Abb. 11 (oben): Blütenstand der Winter-Linde. Abb. 12 (Mitte): Fruchtstand der Winter-Linde. Abb. 13 (unten): Linden-Keimling. 
nen die dünnschaligen Früchte der WinterLinde mit den Fingern zerdrückt werden, die der Sommer-Linde hingegen nicht. Die Früchte sind für viele Tiere eine wichtige Nahrungsquelle. Mäuse verschleppen die Früchte auch in die Nähe von Mauerritzen, sodass selbst in der Stadt oft im weiten Umkreis einer Linde an verschiedensten Standorten Lindenkeimlinge zu finden sind. Die Keimlinge sind für den Ungeübten auf den ersten Blick nicht als junge Linden zu erkennen, da die Keimblätter tief eingeschnitten sind und keinerlei Ähnlichkeit mit der Form der primären und sekundären Laubblätter aufweisen.

\section{Häufig gepflanzte Linden-Arten}

Linden wurden früher in die eigene Familie der Lindengewächse (Tiliaceae) gestellt. Aufgrund neuerer Untersuchungen wurden die Lindengewächse in die Malvengewächse (Malvaceae) integriert und bilden dort die Unterfamilie Tilioideae (BAyER et al. 1998). Weltweit sind 23 Linden-Arten bekannt (Рigott 2002), dazu kommen einige Natur-Hybriden. Sie sind in temperaten Regionen Nordamerikas heimisch und auch bei uns im Freien kultivierbar. Südlich dringen Linden auf dem amerikanischen Kontinent bis nach Mexiko und in Ost-Asien bis in den indochinesischen Raum vor (RolofF \& B ̈̈̈rTELS 1999).

In der Gartenkultur spielen bei uns nur wenige Linden-Arten eine größere Rolle, darunter die beiden in Deutschland heimischen Arten Sommer-Linde (T. platyphyllos) und WinterLinde (T. cordata), vor allem aber die Hybride aus diesen beiden Arten (Tilia $\times$ europaea $=T$. vulgaris, Holländische Linde, Bastard-Linde) sowie die aus Südost-Europa und Kleinasien stammende Silber-Linde (T. tomentosa). Um die vorletzte Jahrhundertwende war es modern, besonders die wegen ihres unterseits silbrigen, attraktiven Laubes sehr geschätzte Silber-Linde

Abb. 14 (oben): Linden treiben an ihren Stümpfen schnell und kräftig wieder aus.

Ab. 15 (Mitte): Knospe der Winter-Linde.

Abb. 16 (unten): Borke der Sommer-Linde.
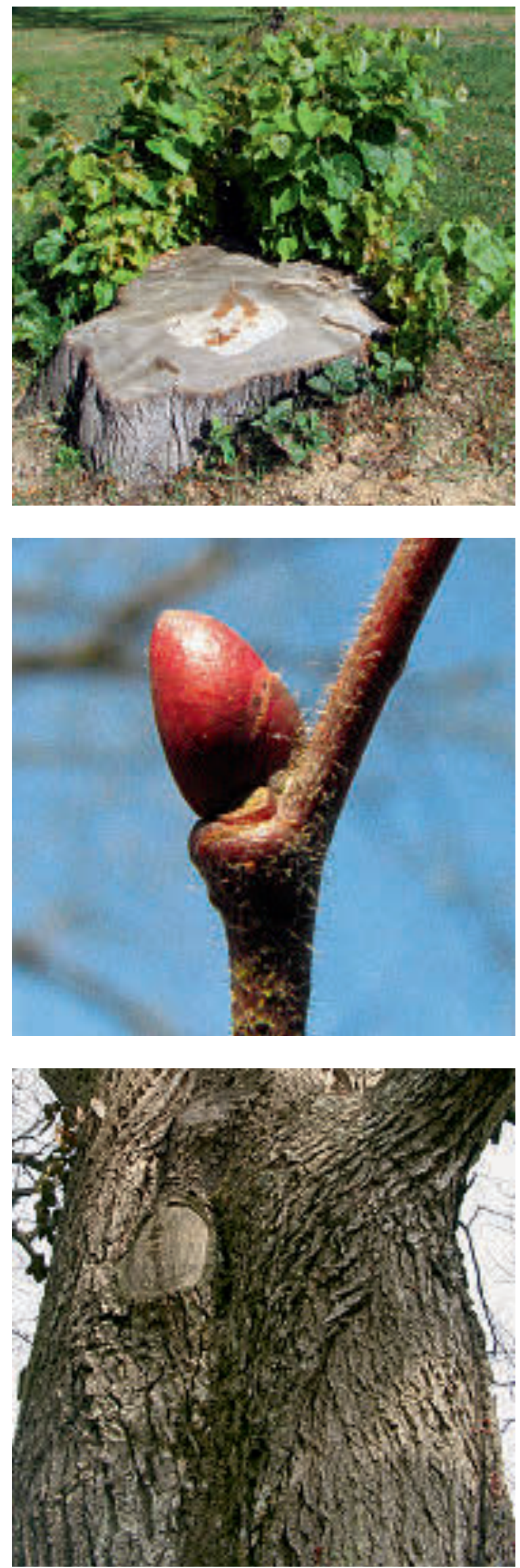

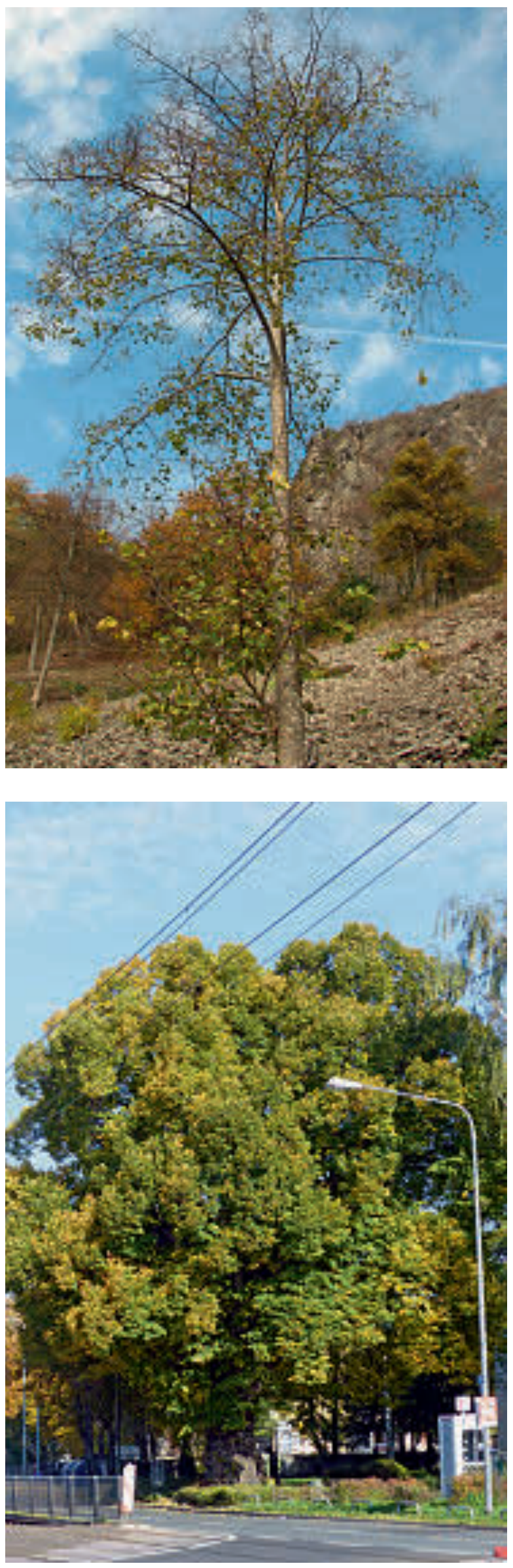

zu pflanzen. In Dortmund beispielsweise wurden in dieser Zeit an vielen Stellen der Stadt Silber-Linden gepflanzt. Noch stehen z. B. einige dieser mittlerweile über 100 Jahre alten prächtigen Stadt-Linden auf dem Anfang des 20 Jh. im Stil eines englischen Landschaftsparks gestalteten Dortmunder Hauptfriedhof. Leider haben Bäume in der Stadt, auch wenn sie kerngesund und über 100 Jahre alt sind, oft keine Überlebenschance, wenn sie Straßenbaumaßnahmen im Wege stehen und dann schnell gefällt werden.

In großen Parkanlagen eignen sich Linden sowohl zur Solitärstellung als auch zur Pflanzung in Gruppen. Besonders für die Holländische Linde sollte viel Platz eingeplant werden, denn sie wird $40 \mathrm{~m}$ hoch und soll dann ihre Elternarten an Schönheit übertreffen (KRÜssMANN 1978). Als Straßenbaum verwendet wird auch die Krim-Linde (T. $x$ euchlora), eine Hybride zwischen $T$. cordata und $T$. dasystyla. Wegen ihrer geringeren Wuchshöhe $(9-15 \mathrm{~m})$ und der späten Blütezeit eignet sich $T$. henryana auch als besonderes Blütengehölz für Gärten. In der freien Landschaft können Linden zur Anlage von Baumhecken genutzt werden. Sommer-, Winter- und Holländische Linden sind Tief- oder Herzwurzler mit einem hohen Anteil von Faserwurzeln im oberen Bodenhorizont und eignen sich daher hervorragend zur Bodenbefestigung. Aufgrund der hohen Ansprüche, die die Arten an den Standort stellen (wärmeliebend, benötigen nährstoffreiche und ausreichend feuchte Böden, reagieren empfindlich gegenüber Luft- und Bodenverschmutzungen) werden sie jedoch nicht als Rohbodenpioniere gepflanzt.

Die Winter-Linde ist ein in Europa weit verbreitetes Gehölz, wobei sie vor allem in den

Abb. 17 (oben): Tilia cordata auf einer Blockschutthalde am Hohentwiel in der Nähe des Bodensees.

Abb. 18 (unten): Die alte Tilia platyphyllos in Frankfurt Eschersheim.

Abb. 19 (Seite 115): Historische Aufnahme der Fahrradbahn im Palmengarten. Rechts im Bild sind die Linden an der Begrenzung des Sportareals zu sehen. 
Mittelgebirgen vorkommt, im Tiefland des Nordens dagegen selten ist. Die Sommer-Linde ist ein in Mittel_ und Südeuropa heimischer Baum, kommt in der Natur aber seltener vor als die Winter-Linde. In Deutschland sind wild wachsende Lindenbestände durch eine jahrhundertelange Nutzung des Holzes selten geworden. Natürliche Standorte der Winter-Linde sind wärmebegünstigte Hang- und Schluchtwälder. Sie kommt aber auch in artenreichen Kiefern- und Lindenmischwäldern sowie in Auwäldern außerhalb des Überschwemmungsbereichs vor. Auch auf Blockschutthalden ist die Winter-Linde anzutreffen. Die Sommer-Linde ist ebenfalls ein typischer Vertreter in Schluchtwäldern, sie ist aber auch in Bergwäldern sowie in wärmeliebenden Laubmischwäldern und auf Geröllhalden anzutreffen (KIERMEIER 1993).

\section{Frankfurter Linden}

Auch Frankfurt ist eine Stadt der Linden. Im Frankfurter Westend gibt es in der Nähe des Palmengartens eine Lindenstraße. Frankfurt hat eine berühmte alte, als Naturdenkmal ausgewiesene Sommer-Linde, nach der auch die Straße Am Lindenbaum sowie die U-BahnHaltestelle Lindenbaum benannt sind. Die alte Frankfurter Linde befindet sich im Stadtteil Eschersheim und wurde um 1700 gepflanzt, damals noch auf freiem Feld als Landmarke. In ihrer Nähe gab es noch eine etwa 50 Jahre ältere Linde, die als große Linde bezeichnete wurde, während die neue dementsprechend die kleine Linde war. Die Reste der großen Linde stürzten im Jahr 1923 um. Die Eschersheimer Linde ist heute rund $20 \mathrm{~m}$ hoch bei einem Stammdurchmesser von $170 \mathrm{~cm}$. Sie ist ein Wahrzeichen der Stadt Frankfurt. Durch den U-Bahn-Bau schien der alte Baum stark gefährdet zu sein, es wurde sogar über die Fällung nachgedacht. Die alte Linde hat glücklicherweise alle Baumaßnahmen gut überstanden und ist vital. Drainage sowie künstliche Bewässerung und Belüftung sorgen für verbesserte Standortbedingungen des Baumes an der verkehrsreichen Eschersheimer Landstraße.

Eine gut $1,5 \mathrm{~km}$ lange Lindenallee wurde Ende der 1980er-Jahre im Zuge der Gestaltung

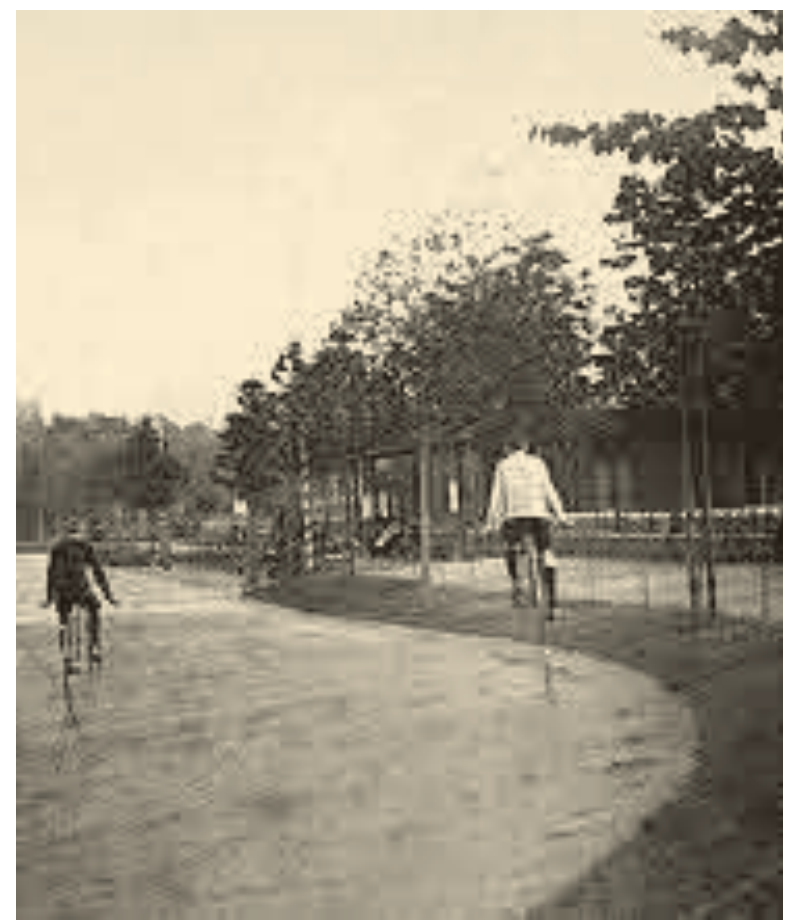

der Bundesgartenschau im Niddapark in Frankfurt Ginnheim gepflanzt. Hier und anderswo kann man regelmäßig an der Basis vieler Lindenstämme Massen der Feuerwanzen (Pyrrhocoris apterus) beobachten. Die Insekten fallen durch ihre markante rot-schwarze Körperzeichnung auf. Feuerwanzen ernähren sich von den heruntergefallenen Früchten der Linde, was ihr bevorzugtes Auftreten an Tilia-Arten erklärt.

Auch der Palmengarten beherbergt noch einige alte Linden, wobei es sich meist um Holländische Linden handelt. Südlich vom Haus Leonhardsbrunn steht eine Krim-Linde (Tilia $x$ euchlora) auf einer kleinen Anhöhe, dem sogenannten Lindenhügel. Der Baum ist ca. 125 Jahre alt und hat eine Blitzrippe von der Krone bis zum Stamm. Diese Linde sowie die großen Exemplare in der Nähe der Steppen- und Spielwiese sind noch Reste der alten Einfassung der ehemaligen Sportanlagen (Fahrrad- und Rollschuhbahn) im Palmengarten. Ebenfalls aus den Anfangszeiten des Palmengartens stammt die etwa 125 Jahre alte Hänge-Silber-Linde ( $T i$ lia tomentosa 'Petiolaris') an den Resten des Brückenkopfes der Hängebrücke über den Großen Weiher. Eine deutlich jüngere Silber-Linde steht schräg gegenüber des Subantarktishauses. 
Markant und schon auf historischen Ansichtskarten abgebildet sind die beiden Alleen aus geschnittenen Holländischen Linden, die links und rechts des Blumenparterres auf das Gesellschaftshaus zuführen. In ihrer Nachbarschaft an der Zufahrt zum Gesellschaftshaus steht eine noch junge Tilia henryana. Seltener in Parks und Gärten zu finden sind die Amerikanische Linde (Tilia americana) an der westlichen Begrenzung des Konzertgartens sowie die Moltke-Linde (Tilia x moltkei) in der Nähe des Birkenhaines. Die Blätter der Amerikanischen Linde sind an ihrer deutlichen, abgesetzten Blattspitze gut zu identifizieren (BEAN 1981). Die Moltke-Linde ist eine Hybride zwischen $T$. americana und T. tomentosa 'Petiolaris', die vor 1880 in der Berliner Baumschule SpÄTH entstanden ist (KRÜSSMANN 1978). Ihre Blätter ähneln denen der Amerikanischen Linde, sind aber wie bei der Silber-Linde unterseits relativ stark behaart.

\section{Linden und das Hummelsterben}

Besonders in Norddeutschland repräsentieren Linden in der Vegetationsperiode meist eine der letzten großen Trachtquellen für Honigbienen und Hummeln (Illies 2005). Unter WinterSommer-, Krim- und Silber-Linden sind zur Blütezeit massenhaft tote Hummeln zu beobachten. Dabei sterben der Tiere relativ rasch. Etwa 45 Minuten nach dem Herabfallen verenden die Tiere in Rückenlage (Surholt et al. 1992). In den vergangenen Jahren geriet dadurch besonders die Silber-Linde in den Verruf, durch die Produktion von giftigem Nektar für das massenhafte Sterben von Hummeln, die sich am Nektar verköstigt hatten, verantwortlich zu sein, wodurch Linden rasch als hymenopterenfeindliche Gehölze eingestuft wurden. Teilweise wurden sogar Empfehlungen ausgesprochen, weniger Linden zu pflanzen oder gar Altbäume zu fällen (Rотн et al. 1994).

Jedoch konnten in den Untersuchungen von
Surholt et al. (1992) sammelten unter Silber-Linden in Rückenlage liegende, noch lebende Hummeln ein und setzten diese wieder auf Blüten der Silber-Linden, welche am Tag zuvor vor Absammlung durch andere nektarsammelnde Insekten mittels Gazebeutel geschützt wurden. Die Hummeln begannen nach kurzer Zeit mit der Nektaraufnahme, sodass die Tiere bereits nach 15-20 Minuten wieder aktiv wurden, weiterhin Nektar tranken und nach rund 30-45 Minuten wieder völlig vital waren. Auch dieses Experiment zeigt deutlich, dass der von Linden sekretierte Nektar weder akut noch längerfristig toxisch für Hummeln ist (SURHOLT et al. 1992). Surholt \& BaAL (1995) nennen zwei mögliche Theorien zum Hymenopterensterben unter Linden. Viele Linden repräsentieren Duftfallen für Insekten. Besonders die spätblühenden Linden-Arten (darunter vor allem die Silber-Linde) verströmen einen intensiven weitstreichenden süßen Duft, der viele Bestäuber anlockt, jedoch enthalten die Blüten nur wenig oder keinen Nektar. Im
Umfeld aufgeblühter Silber- oder Krim-Linden gibt es zu diesem Zeitpunkt nur noch wenige andere blühende Trachtpflanzen, sodass Insekten quasi zum Besuch der Lindenblüten gezwungen werden. An den Bäumen entsteht Nahrungskonkurrenz um den wenig vorhandenen Nektar. Dabei wenden die Insekten wesentlich mehr Energie für die Nektarsuche auf, als letztendlich aufgenommen wird. Aufgrund dieses stetig größer werdenden Energiedefizits werden die Blütenbesucher flugunfähig, fallen vom Baum herab, kühlen auf dem Boden aus und sterben dort letztendlich (Surholt \& BaAl 1995). Die Silber-Linde blüht in einem Zeitraum von etwa 15 Tagen. Dabei wird jedoch nicht kontinuierlich gleich viel Nektar produziert. Messbare Nektarproduktionen sind nur vom 1. bis zum Nachmittag des 6. Tages nachweisbar, wobei das Maximum der Nektarproduktion am 6. Tag liegt. Ab dann sinkt die Nektarproduktion rapide auf ein verschwindend geringes Maß (Surholt \& BAAL 1995). 
Denker et al. (1992) nur Fructose, Saccharose und Glukose im Nektar von Silber-Linden nachgewiesen werden, giftige Mannose oder ein anderes toxisches Mono- oder Disaccharid hingegen nicht. Ein ähnliches Bild zeigte sich auch für den Nektar von Winter- SommerKrim- und Silber-Linden, in dem ebenfalls weder Mannose noch andere giftige Mono- oder Disaccharide vorhanden sind (Denker et al. 1992, BaAl et al. 1992, 1994). Auch in den Körpern geschädigter Hummeln, die zuvor Lindennektar aufgenommen hatten, wurden weder Mannose noch ein anderes giftiges Mono- und Disaccharid nachgewiesen (BAAL et al. 1992, 1994). Die Versuche von BaAl et al. (1992), bei denen Hummeln ausschließlich mit Nektar von Silber-Linden über 5 Tage hinweg gefüttert wurden, zeigten eindeutig, dass diese Tiere keinerlei Vitalitätsverlust gegenüber den Tieren des Kontrollversuchs, die über den gleichen Zeitraum mit einer Zuckerlösung ernährt wurden, zeigten. Beide Gruppen wiesen die gleiche Lebensdauer auf.

Das unter Linden häufig zu beobachtende Sterben von Bienen und Hummeln ist also eine Folge des vielfach vorherrschenden Nahrungsmangels im Umfeld von blühenden Linden sowie der geringen Nektarproduktion in den Lindenblüten und nicht eine Folge einer möglichen Vergiftung mit schädlichen Zuckern, wie dies früher vielfach vermutet wurde (BAAL et al. 1994, 1995, Surholt \& BaAl 1995).

Auffällig ist, dass unter Linden vor allem Hummeln, aber vergleichsweise wenige tote Honigbienen zu finden sind. Es konnte nachgewiesen werden, dass es gravierende Unterschiede im Sammelverhalten zwischen Honigbienen und Hummeln gibt (Illies 2005). So sammeln z. B. Honigbienen Lindennektar nur an Tagen hoher Nektarproduktion, da sie offensichtlich über die Fähigkeit verfügen, die Qualität des Lindennektars zu bewerten. So kann sicherge-

Abb. 20 (oben): 125-jährige Krim-Linde auf dem Lindenhügel im Palmengarten.

Abb. 21 (Mitte): Tilia henryana im Palmengarten.

Abb. 22 (unten): Silber-Linde im Herbstaspekt
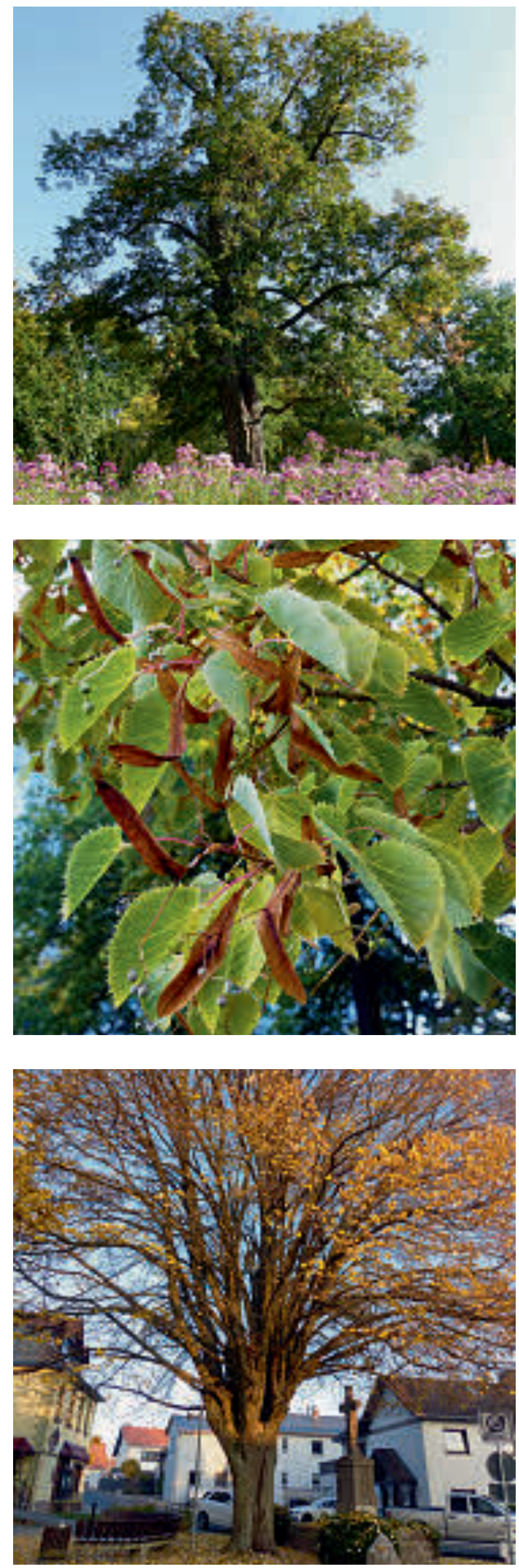


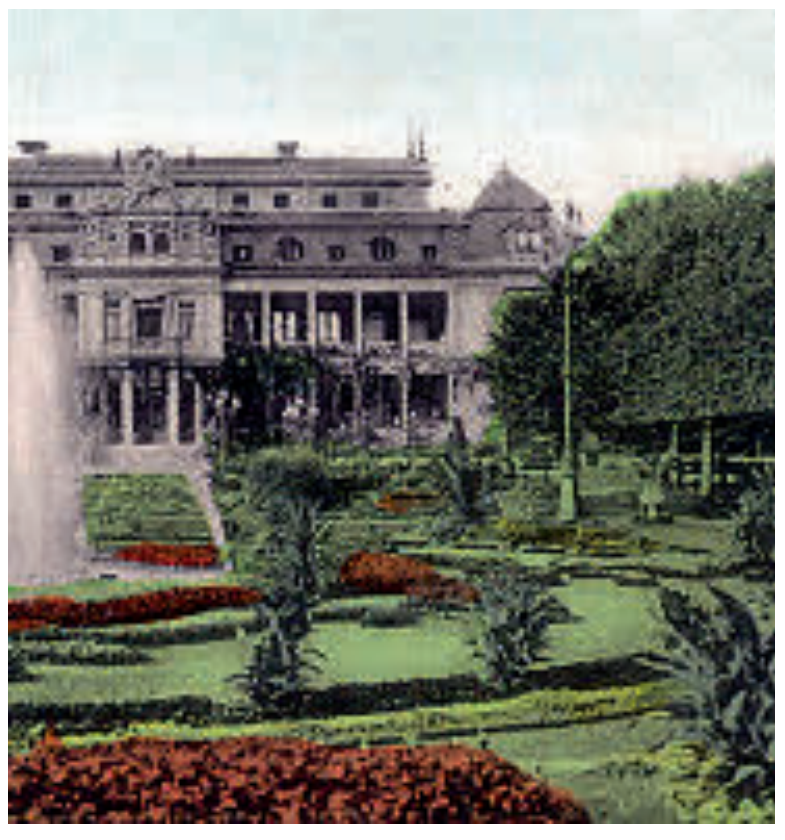

stellt werden, dass die Kosten für den Sammelflug durch energiereichen Nektar ausgeglichen werden und ein Energiedefizit vermieden wird. Zudem sind Honigbienen durch ihre Tanzsprache in der Lage, über das Nektarangebot mit Artgenossen zu kommunizieren. Die Untersuchungen von Illies (2005) haben für Hummeln gezeigt, dass hier im Unterschied zu Honigbienen keine erkennbaren Korrelationen zwischen Sammelaktivität und Nektarverfügbarkeit an Linden besteht. So werden SilberLinden auch noch von Hummeln angeflogen, wenn nur noch wenig Nektar zur Verfügung steht.

\section{Dank}

Herzlicher Dank geht an Bernd Grünewald (Institut für Bienenkunde, Oberusel) für Hinweise zum Bienensterben und David Gustav (Universität Konstanz) für die Unterstützung bei der Literaturrecherche.

\section{Literatur}

Baal, T., Denker, B., Mühlen, W. \& Surholt, B. 1994: Die Ursache des Massensterbens von Hummeln unter spätblühenden Linden. - NuL 69: 412-418.

BaAl, T., Denker, B., Mühlen, W., Riedel, V. \& Surholt, B. 1992: Zusammensetzung der Zucker im Körper von Hummeln, die Lindenblüten besuchen. Apidologie 23: 333-335.

Bayer, C., Fay, M. F., Bruijn, A.Y. de, Savolainen, V.,
Morton, C. M., Kubitzki, K., Alverson, W. S. \& Chase, M. W. 1999: Support for an expanded family concept of Malvaceae within a recircumscribed order Malvales: a combined analysis of plastid $a t p \mathrm{~B}$ and $r b c \mathrm{~L}$ DNA sequences. - Bot. J. Linn. Soc. 129: 267-303.

BEAN, W. J. 1981: Trees and shrubs hardy in the British isles. 8. Aufl. - London.

BlascheK, W. 2007 (Hrsg.): Hagers Enzyklopädie der Arzneistoffe und Drogen. - Stuttgart.

Denker, B., Bafl, T., Mühlen, W., Popp, M., Riedel, V. \& Surholt, B. 1992: Zuckerspektrum des Nektars spätblühender Linden. - Apidologie 23:331-329.

Düll, R. \& Kutzelnigg, H. 2011: Taschenlexikon der Pflanzen Deutschlands und angrenzender Länder. Wiebelsheim.

Fischer-Rizzi, S. 1996: Blätter von Bäumen. Legenden, Mythen, Heilanwendungen und Betrachtung von einheimischen Bäumen. - München.

FröHLICH, H. J. 1989: Alte liebenswerte Bäume in Deutschland. - Hamburg.

ILliEs, I. 2005: Verhaltensbiologische Untersuchungen zur Trachtnutzung und zum Sammelverhalten von Bienen (Hymenoptera, Apoidea). - Dissertation Ruhr-Universität Bochum.

Jablonski, E. \& Plietzsch, A. 2013: Kultivierte Linden, II: Sorten von Tilia cordata Mill., T. platyphyllos Scop. und T. xeuropaea L. - Mitt. Deutsch. Dendrol. Ges. 98: 89-110.

Jablonski, E. \& Plietzsch, A. 2014: Kultivierte Linden, III: Sorten eurasischer und amerikanischer Tilia-Arten und -Hybriden (exkl. T. cordata MiLl., T. platyphyllos Scop. und T. x europaea L.). - Mitt. Deutsch. Dendrol. Ges. 99: 83-102.

JablOnski, E. 2011: Kultivierte Linden (Tilia L., Malvaceae Juss.) in Mitteleuropa I. Arten. - Mitt. Deutsch. Dendrol. Ges. 96: 33-56.

Kiermeier, P. 1993: Wildgehölze des mitteleuropäischen Raumes. BdB-Handbuch, Teil VIII, 5. Aufl. - Pinneberg. Krüssmann, G. I978: Handbuch der Laubgehölze. Band 3. - Berlin, Hamburg.

Kühn, S., Ullrich, B. \& KüHn, U. 2004: Deutschlands alte Bäume. - München.

Laudert, D. 2004: Mythos Baum. - München. Marzell, H. 1979: Wörterbuch der deutschen Pflanzennamen. - Stuttgart.

Abb. 23 (oben): Ausschnitt aus einer alten Ansichtskarte (abgeschickt 1903), die das Gesellschaftshaus mit Blumenparterre und Lindenallee zeigt.

Abb. 24 (Seite 119 oben links): Alte, hohle Linde in der Gegend von Jena.

Abb. 25 (Seite 119 oben rechts): Blick von unten in die herbstliche Allee aus Holländischer Linde vor dem Gesellschaftshaus.

Abb. 26 (Seite 119 unten): Feuerwanze am Stamm einer Holländischen Linde im Palmengarten. 


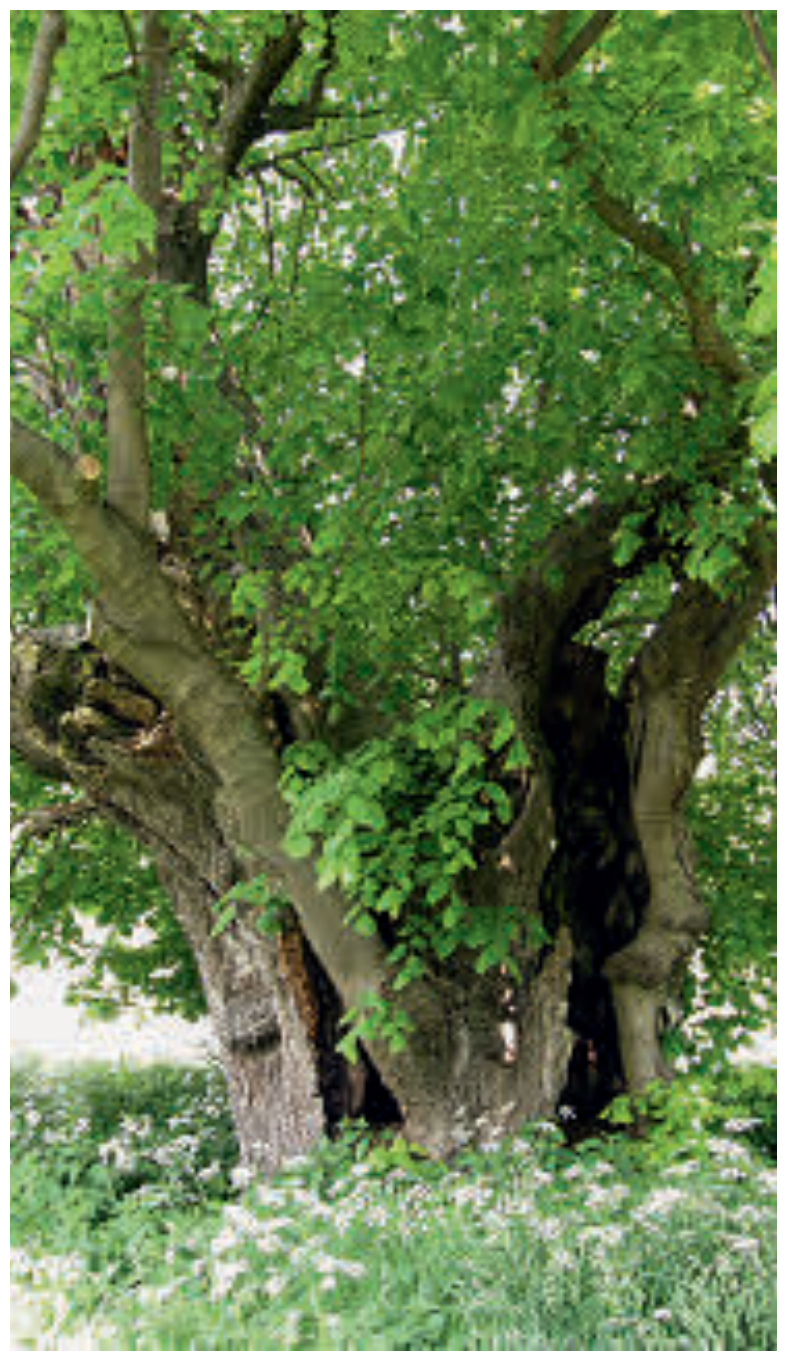

Pigott, C. D. 2002: A review of chromosome numbers in the genus Tilia (Tiliaceae). - Edinb. J. Bot. 59: 239-246. Roloff, A. \& Bärtels, A. 1996: Gartenflora. Bd. 1. Gehölze. - Stuttgart.

Roth, L., Daunderer, M. \& Kormann, M. 1994: Giftpflanzen-Pflanzengifte, 4. Aufl. - Hamburg. Schneebeli-Graf, R. 2008: Die Linde. Ihre Geschichte und Geschichten. Ein botanisch-kulturhistorischer Essay. - Thun.

Sснӥтt, P., Sснucк, H. J. \& Sтімm, B. 2002: Lexikon der Baum- und Straucharten. - Hamburg.

Surholt B. \& BaAl, T. 1995: Die Bedeutung blühender Silberlinden für Insekten im Hochsommer. - NuL 70: 252-258.

Surholt, B., Denker, B., Baal, T. \& Mühlen, W. 1992: Ist Silberlindennektar für Hummeln giftig? Ein VideoProtokoll von Freilandexperimenten. - Apidologie 23: 335-337.

\section{Internetseiten}

http://www.ddg-web.de/index.php/rekordbaeume.html http://www.frankfurt.de/sixcms/detail.php?id=3844\&_ ffmpar\%5B_id_inhalt $\% 5 \mathrm{D}=30398$

https://de.wikipedia.org/wiki/Eschersheimer_Linde
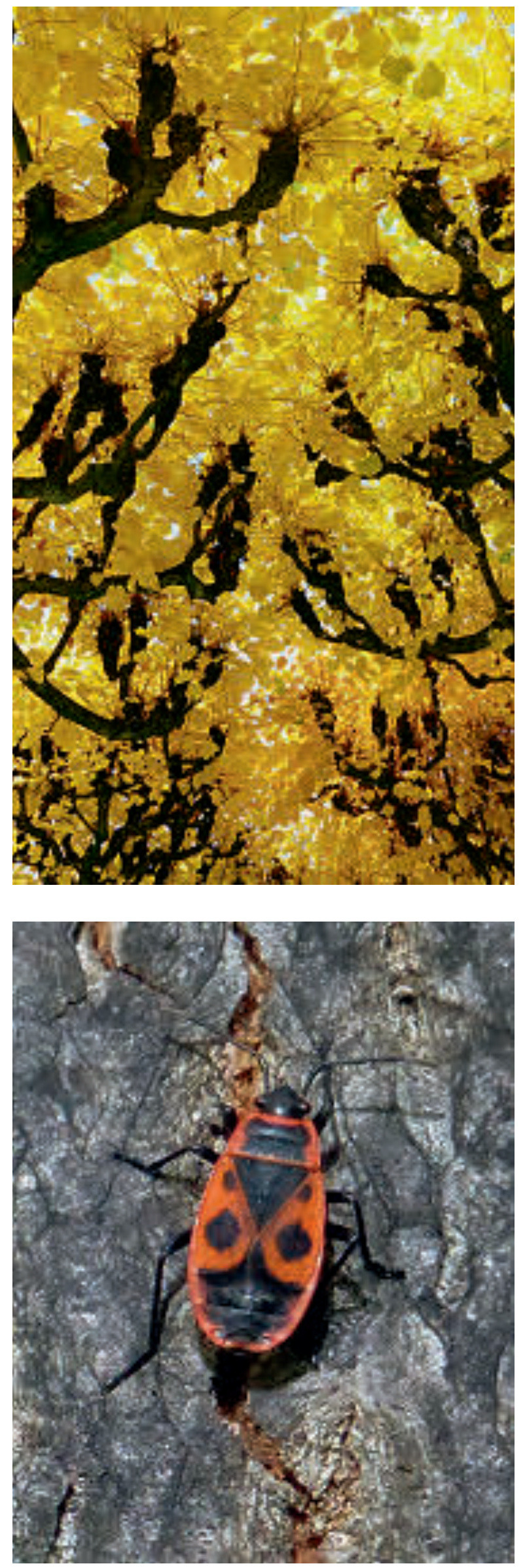